\title{
Comparison of the Minimum Plateau Width by Plateau and a New Method in People with Conductive Hearing Loss
}

\author{
Seyyed Jalal Sameni ${ }^{1}$, Ahmad Daneshi ${ }^{2}$, Akram Pourbakht ${ }^{1}$, \\ Aliakbar Tahaei ${ }^{1}$, and Mohammad Kamali ${ }^{3}$ \\ ${ }^{1}$ Departments of Audiology, ${ }^{3}$ Rehabilitation Management, School of Rehabilitation Sciences, Iran University of Medical Sciences, Tehran, Iran \\ ${ }^{2}$ Research Center of Otolaryngology, Head and Neck Surgery, Hazrat-e-Rasool Hospital, Iran University of Medical Sciences, Tehran, Iran
}

Received March 9, 2018

Revised May 6, 2018

Accepted May 8, 2018

\author{
Address for correspondence \\ Akram Pourbakht, MD, PhD \\ Department of Audiology, \\ School of Rehabilitation Sciences, \\ Iran University of Medical Sciences, \\ Madadkaran St., Shah-Nazari St., \\ Madar Sq., Mirdamad Blvd., \\ Tehran 1545913187, Iran \\ Tel +98-21-2222-8051 \\ Fax +98-21-2222-0946 \\ E-mail Pourbakht.a@iums.ac.ir
}

Background and Objectives: In clinical masking, the plateau is a state in which the non-test ear (NTE) is completely masked by the noise and tone is heard only by the test ear (TE). At least 15 to $20 \mathrm{~dB}$ of plateau width is needed to obtain valid threshold. In the study, a part of plateau after initial masking level known as the minimum plateau width (mPW) was determined and compared by a new formula and the plateau searching method. Subjects and Methods: Minimum plateau widths of air conduction were obtained in 29 participants with unilateral and 30 participants with bilateral conductive hearing loss $(\mathrm{CHL})$ aged 20 to 45 years old by using step by step plateau method and mPW estimation by the formula between two points of masking diagram $\left[\mathrm{mPW}=\left(\mathrm{N}_{2}-\mathrm{N}_{1}\right)-\left(\mathrm{T}_{2}-\mathrm{T}_{1}\right)\right]$ and then the $\mathrm{mPW}$ of two methods was compared for each frequency. Results: There was no significant difference between the minimum plateau width obtained by the plateau and formula methods for two given point of masking diagram in people with unilateral and bilateral $\mathrm{CHL}$ at octave frequencies from $500 \mathrm{~Hz}$ to $4,000 \mathrm{~Hz}$. Conclusions: Threshold obtaining of TE by two tones for two noise levels delivered to the NTE is enough to estimate the MPW between these two noise points and it is not necessary that for clinicians to know the actual values of masking diagram components.

J Audiol Otol 2018;22(4):229-235

\section{Introduction}

Pure tone audiometry at octave frequencies from $250 \mathrm{~Hz}$ to $8,000 \mathrm{~Hz}$ is a basic test of hearing that was developed some time ago. In this test, both air and bone conduction thresholds are obtained for each ear, and obtaining the actual threshold for each ear is very important. There are situations in which the patient's response is not related to the test ear (TE) but instead is due to contributions from the opposite ear [non-test ear (NTE)]. This phenomenon occurs when the pure tone level is high enough to reach the other ear's bone threshold. Of course, the signal detected by the NTE is lower than the signal level presented to the TE. This energy loss from one ear to another is called interaural attenuation (IA) [1].

This is an Open Access article distributed under the terms of the Creative Commons Attribution Non-Commercial License (https://creativecommons.org/licenses/by-nc/4.0/) which permits unrestricted non-commercial use, distribution, and reproduction in any medium, provided the original work is properly cited.
Clinical masking procedures should be used in which a noise regularly presented to NTE to avoid signal being heard in that ear. This seems simple, though as Valente [2] pointed out in her book (p.85) "clinical masking in audiology is one of the most difficult clinical concepts for the student of audiology to master," and it needs basic knowledge about cross hearing, that is, the perception of sound in one ear that has crossed over the head via bone transmission, presented through an earphone to opposite ear [3]. Without adequate masking of the NTE, hearing thresholds in the TE may be false and lead to misinterpretation of the type of hearing loss [2].

There are various methods for clinical masking that can be divided into two methods: acoustic (i.e., formula) and psychoacoustic methods [4]. In the acoustic method, the amount of masking is based on the approximate calculation of the test signals and masker levels in each ear. For example, Liden, et al.'s equations [5] can be used to determine the minimum effective and maximum usable masking for air and bone 
conductions. These formulas for air conduction (AC) and bone conduction (BC) testing include:

$$
\begin{aligned}
& \mathrm{MEM}_{\mathrm{AC}}=\mathrm{AC}_{\mathrm{TE}}+\mathrm{ABG}_{\mathrm{NTE}} \mathrm{IA}, \\
& \mathrm{XUM}_{\mathrm{AC}}=\mathrm{BC}_{\mathrm{TE}}+\mathrm{IA}, \\
& \mathrm{MEM}_{\mathrm{BC}}=\mathrm{BC}_{\mathrm{TE}}+\mathrm{ABG}_{\mathrm{NTE}}, \\
& \mathrm{XUM}_{\mathrm{BC}}=\mathrm{BC}_{\mathrm{TE}}+\mathrm{IA},
\end{aligned}
$$

where $\mathrm{MEM}_{\mathrm{AC} \& \mathrm{BC}}$ are the minimum effective masking levels for $\mathrm{AC}$ and $\mathrm{BC}$, respectively, $\mathrm{XUM}_{\mathrm{AC}} \& \mathrm{BC}$ are the maximum usable masking levels for $\mathrm{AC}$ and $\mathrm{BC}$, respectively, $\mathrm{AC}_{\mathrm{TE}}$ is the actual $\mathrm{AC}$ threshold of $\mathrm{TE}, \mathrm{ABG}_{\mathrm{NTE}}$ is the actual air-bone gap (ABG) in the NTE, $\mathrm{BC}_{\mathrm{TE}}$ is the actual $\mathrm{BC}$ threshold in the TE, and IA is the actual interaural attenuation for AC [6]. By using these formulas, everyone can calculate the overall plateau width for the actual $\mathrm{AC}$ and $\mathrm{BC}$ thresholds of TE, $\mathrm{ABG}_{\mathrm{NTE}}$ and actual value of IA at each test frequency.

According to Saunders [7], when using the psychoacoustic method, we should consider plateau tracking, NTE threshold shifting, and shadow responses, which are more acceptable in clinical audiometry. Hood [8] near to six decades ago in 1960 introduced a psychoacoustic method that later became known as the plateau method. In this procedure, after determining the necessity of masking, a noise (masker) at the initial level is presented to NTE and the TE threshold reestablished. As the TE threshold changes, the noise level (in the NTE) and tone level (in the TE) is increased in steps until further increases in the noise level produce no further increases in the tone level. Then, the actual threshold of the TE is obtained at the plateau part of masking diagram [8].

The masking process is diagramed in Fig. 1; it ideally contains three parts: undermasking, plateau or effective masking, and overmasking. In all three parts, masking is done. In the undermasking, in spite of noise presentation to the NTE, the tone delivered to the TE still is heard in the NTE. In the plateau, the tone level has reached the TE threshold, which can be determined by raising noise level in the NTE and noting there is no change in the threshold of the tone. In overmasking, the noise level presented to NTE is so high that it crosses to the TE and as the noise increases, the tone also increases [9].

According to the above description, any level of noise in the plateau section is sufficient for clinical masking. Therefore, the plateau width is important during masking. Martin and Clark [1] have stated that the plateau width is determined by three factors: 1) the AC threshold of the NTE, 2) the BC threshold of the TE, and 3) subject's IA. By decreasing the AC threshold of the NTE, increasing the BC threshold of the TE and with larger IA, the plateau width increases and vice versa [1].
Considering the importance of plateau width and by using the geometric structure of the masking diagram, we introduced a new formula for estimating part of the plateau between the two points of this diagram. Based on the sections characteristics of the masking diagram in Fig. 2, the horizontal line represents the plateau (constant tone thresholds) while the diagonal lines represent the undermasking and overmasking parts. In these later parts, noise and tone changes are in the same value (accepting the condition of effective masking), therefore virtually removing the plateau part, which leads to the direction of undermasking and overmasking at the same line. This means that noise differences be-

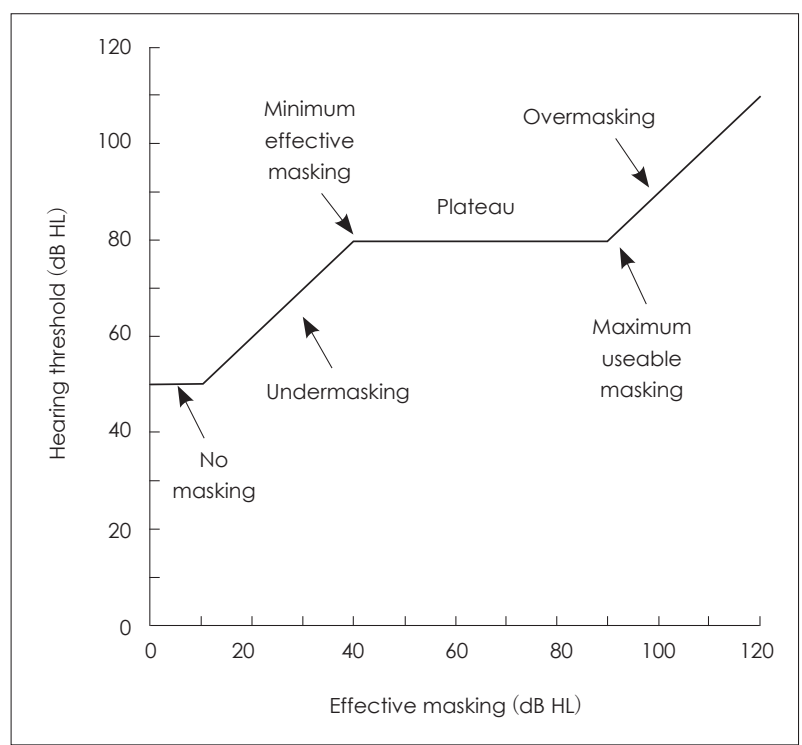

Fig. 1. Hypothetical example illustrating the concepts of undermasking, plateau, and overmasking using the plateau masking procedure.

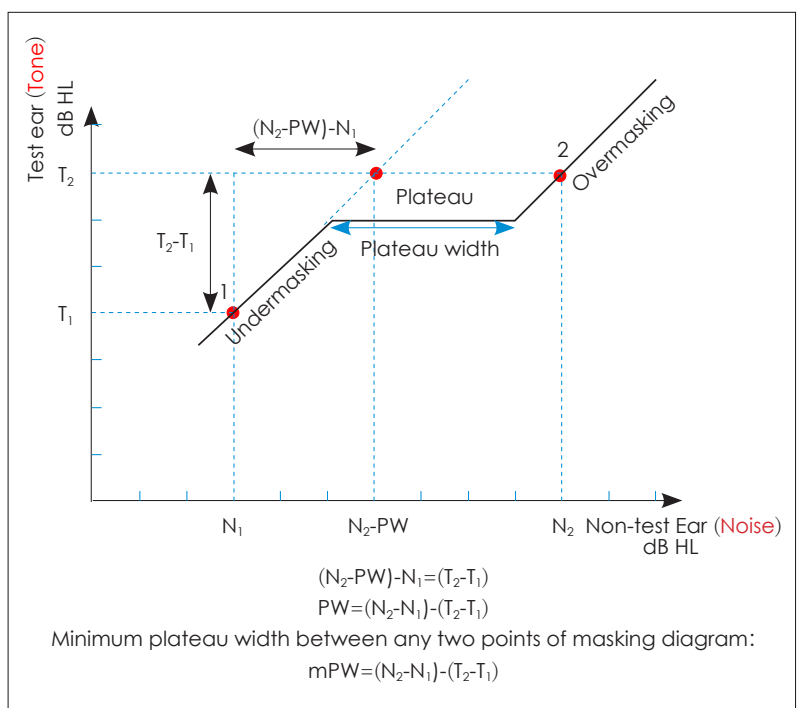

Fig. 2. Schematic masking diagram. 
tween any two points $\left(\mathrm{N}_{2}\right.$ and $\left.\mathrm{N}_{1}\right)$ are equal to tones difference between them $\left(T_{2}\right.$ and $\left.T_{1}\right)$. As a result, the plateau width (PW) can be calculated between these two points according to the following equation:

$$
\begin{aligned}
& \left(\mathrm{N}_{2}-\mathrm{PW}\right)-\mathrm{N}_{1}=\left(\mathrm{T}_{2}-\mathrm{T}_{1}\right), \\
& \mathrm{PW}=\left(\mathrm{N}_{2}-\mathrm{N}_{1}\right)-\left(\mathrm{T}_{2}-\mathrm{T}_{1}\right) .
\end{aligned}
$$

Because the $\mathrm{N}_{1}$ and $\mathrm{N}_{2}$ can be located in the plateau section of the masking diagram, the width of the plateau segment between these two points is less than the overall plateau width, and as a result the minimum plateau width is replaced by plateau width:

$$
\mathrm{mPW}=\left(\mathrm{N}_{2}-\mathrm{N}_{1}\right)-\left(\mathrm{T}_{2}-\mathrm{T}_{1}\right)
$$

where $\mathrm{mPW}$ is the minimum plateau width between these two points (2 and 1) as shown on the schematic masking diagram. $\mathrm{N}_{2}$ and $\mathrm{N}_{1}$ are the noise levels (in $\mathrm{dB} \mathrm{HL}$ ) at point 2 and 1 , respectively, on the $\mathrm{X}$-axis of the diagram, and $\mathrm{T}_{2}$ and $\mathrm{T}_{1}$ are the pure tone levels (in $\mathrm{dB} H \mathrm{HL}$ ) at point 2 and 1, respectively, on the Y-axis of the diagram.

Since the $\mathrm{mPW}$ equation was extracted from the masking diagram, it can be extrapolated to all audiograms. As previously mentioned, plateau width is affected by ABG, and according to Turner $[6,10]$, the AC plateau width can be computed by the following equation:

$$
\mathrm{PW}_{\mathrm{AC}}=2 \mathrm{IA}-\mathrm{ABG}_{\mathrm{TE}}-\mathrm{ABG}_{\mathrm{NTE}} \text {, }
$$

where $\mathrm{PW}_{\mathrm{AC}}$ is the plateau width for the $\mathrm{AC}$ testing, IA is the actual interaural attenuation, and $\mathrm{ABG}_{\mathrm{TE}}$ and $\mathrm{ABG}_{\mathrm{NTE}}$ are the actual air-bone gap for TE and NTE, respectively.

In respect to Turner's equation, this survey focused on unilateral conductive hearing loss $(\mathrm{CHL})$, in which $\mathrm{ABG}_{\mathrm{NTE}}$ is zero, and initial bilateral $\mathrm{CHL}$ that the plateau width is narrower.

As mentioned, estimation of a mPW can be helpful for clinicians before performing the clinical masking. Because of the importance of masking applications in clinical evaluations of hearing and the role of the applicable mPW, the aim of this study was to estimate the $\mathrm{mPW}$ by our $\mathrm{mPW}$ formula and compare with the $\mathrm{mPW}$ that was obtained by the plateau method in people with unilateral and bilateral CHL.

\section{Subjects and Methods}

\section{Participants}

This survey was carried out in the Audiology Clinic, School of Rehabilitation Sciences, Iran University of Medical Sciences. Using Turner's equation: $\left(\mathrm{PW}_{\mathrm{AC}}=2 \mathrm{IA}-\mathrm{ABG}_{\mathrm{TE}}-\mathrm{AB}-\right.$ $\mathrm{G}_{\mathrm{NTE}}$ ), we conducted this research on persons with CHL (unilateral and bilateral). Two groups of adult subjects with unilateral and bilateral CHL, 20-45 years old, participated in this study: 29 subjects with unilateral CHL (12 men and 17 women) with a mean age and standard deviation of $34.14 \pm 5.42$ years, and 30 subjects with bilateral CHL (23 men and 7 women) with a mean age and standard deviation of $38.13 \pm 5.03$ years. These people were selected after performing otoscopy, immitance audiometry, and $\mathrm{AC}$ and $\mathrm{BC}$ pure tone audiometry. The experiment was approved by the Research Ethics Committee of Iran University of Medical Sciences (IR.IUMS.REC 1395. 9211303207). Informed consent letter were obtained for all participants.

To be confident about the possibility of performing masking in the unilateral CHL group, their hearing losses were proven by using the plateau method. However, in bilateral CHL group, due to the presence of significant $\mathrm{ABG}$ in the NTE in some people, it was not possible to mask it, which had no effect on the implementation of the study. To eliminate the influence of factors such as occlusion effect and bone vibrator displacement during the test, $\mathrm{mPW}$ was determined for $\mathrm{AC}$. For this reason, individuals with $\mathrm{AC}_{\mathrm{TE}}-\mathrm{BC}_{\mathrm{NTE}} \geq 40 \mathrm{~dB}$ at most frequencies were enrolled. In these groups, $\mathrm{BC}$ thresholds were normal in both ears before masking.

\section{Equipment}

The pure tone audiometry was performed by a Madsen Orbiter 922 audiometer (Otometrics, Natus Medical Inc., Taastrup, Denmark) with TDH39 Circumaural earphones and a B-71 bone vibrator for obtaining $\mathrm{AC}$ and $\mathrm{BC}$ thresholds, respectively. Immitance audiometry including tympanometry and reflexometry was performed with a Madsen Zodiac 901 middle ear analyzer (Otometrics, Natus Medical Inc.).

\section{Experimental procedures}

After recruiting participants, loudness discomfort levels (LDL) were obtained by narrow band noise at 500, 1,000, 2,000 , and 4,000 Hz. In the next step, the mPW between initial masking level (IML) and $10 \mathrm{~dB}$ less than LDL were determined by: 1) the formula method and then 2) the plateau searching method. Several break times were considered during the test to reducing the fatigue of subjects.

Since the maximum output of narrow band noise (NBN) in Orbiter 922 is $105 \mathrm{~dB} \mathrm{HL}$ at $500 \mathrm{~Hz}$ and $110 \mathrm{~dB} \mathrm{HL}$ at $1,000 \mathrm{~Hz}$ to $4,000 \mathrm{~Hz}$, whenever the LDL of noise was greater than the audiometer maximum output, $\mathrm{N}_{2}$ was considered equal to $100 \mathrm{~dB} \mathrm{HL}$. 
To run the masking for AC pure tone audiometry, a biological calibration was performed for narrow band noises in the frequencies of 500, 1,000, 2,000, and 4,000 Hz. This calibration was carried out for 12 normal hearing listeners using the method mentioned by Martin and Clark [1].

Formula method:

1) The TE thresholds were measured after NBN was delivered at $\mathrm{AC}_{\mathrm{NTE}}+15 \mathrm{~dB}$ (the IML) in the NTE to determine $\mathrm{N}_{1}$ and $T_{1}$ (The IML of this survey was selected according to Gelfand [9]).

2) Again by presenting $N B N$ at $L_{\text {DLLE }}-10 \mathrm{~dB}$ (or $100 \mathrm{~dB}$ $\mathrm{HL}$ ), TE thresholds were obtained to get $\mathrm{N}_{2}$ and $\mathrm{T}_{2}$.

3) Thereafter, mPW were computed by $\left(\mathrm{N}_{2}-\mathrm{N}_{1}\right)-\left(\mathrm{T}_{2}-\mathrm{T}_{1}\right)$, individually at $500 \mathrm{~Hz}$ to $4,000 \mathrm{~Hz}$.

Plateau search method:

1) The TE thresholds were measured after NBN was delivered at $\mathrm{AC}_{\mathrm{NTE}}+15 \mathrm{~dB}$ (IML) in the NTE to determine the start point in the plateau curve.

2) By increasing NBN in $5 \mathrm{~dB}$ steps, TE thresholds were retested until reaching the end of plateau section or $\mathrm{LDL}_{\mathrm{NTE}^{-}}$ $10 \mathrm{~dB}$ (or $100 \mathrm{~dB}$ HL). To determine the end of plateau part, this process was continued until at least with two steps of noise increment at NTE and the TE threshold also increased by two steps.

3) To reduce the test error, the above steps were repeated several times until at least two identical responses were achieved.

4) By reducing the noise level at end of plateau part, if possible, from IML in the NTE, the MPW were determined.

In the study, to avoid data bias, the formula method was applied before the plateau search method. The average test time with respect to an individual's cooperation for each frequency was about 3 minutes for the formula method and about 12 minutes for the plateau searching method; and in general, the formula method took 15 minutes and the plateau method took about an hour including the break times. And anybody who were not able to continue the experiment, were excluded.

\section{Statistical analysis}

The statistical analysis was performed by SPSS software ver. 16 (SPSS Inc., Chicago, IL, USA) and $p<0.05$ was considered to be a significant difference in this survey. With respect to the normal distribution of $\mathrm{mPW}$ data by Kolmogorov-Smirnov test, an independent sample t-test was performed to compare the mPWs in the two methods.

\section{Results}

Frequency distribution of participants in the study was almost equal in two groups (49.15\% unilateral CHL \& 51.85\% bilateral $\mathrm{CHL}$ ), but there were more women in the unilateral CHL group (17 vs. 12 men), but more men in bilateral CHL group ( 23 vs. 7 women). The averages of the ABG at the different frequencies for these two groups also are shown in Fig. 3.

The mPW was evaluated at first by the formula and then by the plateau search method at different frequencies in each group. The average and SD for $\mathrm{mPW}$ of the two groups are shown in Table 1 for methods and frequencies, separately. As shown, the means of $\mathrm{mPW}$ for the bilateral CHL group are lower than for the unilateral CHL group. This is due to different ABGs from 500 to $4,000 \mathrm{~Hz}$ in the NTE. This is also
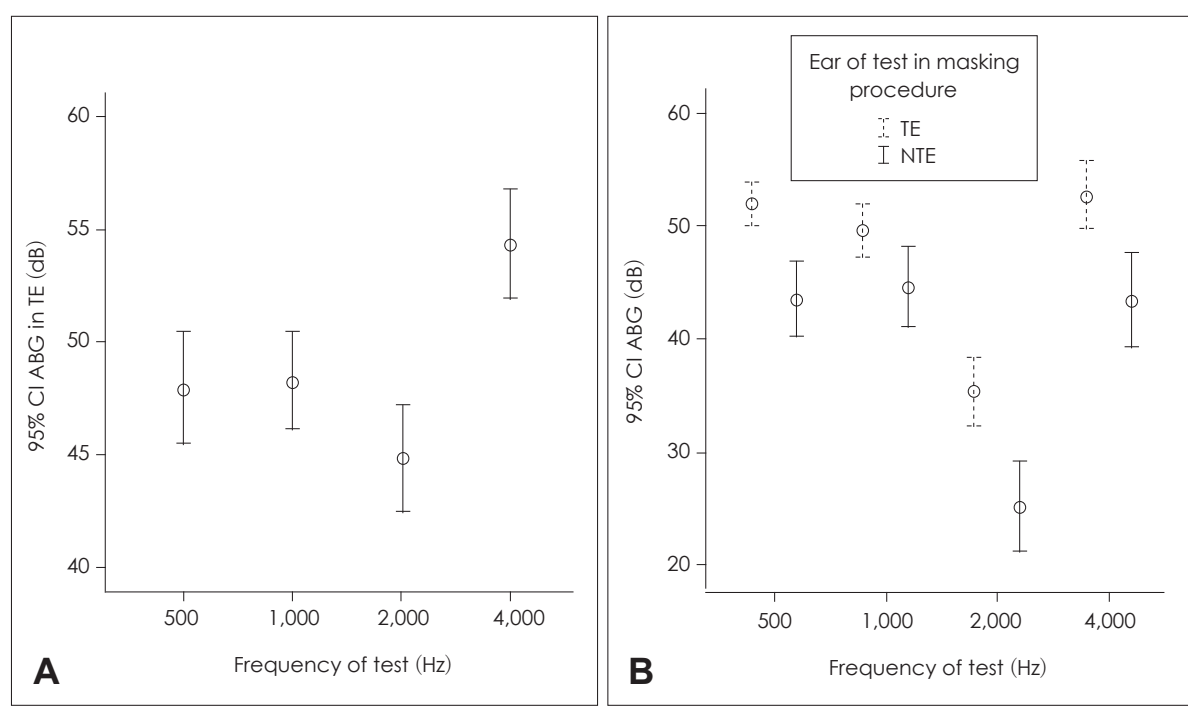

Fig. 3. Average of $A B G$ at $500 \mathrm{~Hz}$ to $4,000 \mathrm{~Hz}$. A: Unilateral $\mathrm{CHL}$ group. $\mathrm{B}$ : Bilateral $\mathrm{CHL}$ group for TE and NTE. Cl: confidence interval, ABG: air bone gap, $\mathrm{CHL}$ : conductive hearing loss, TE: test ear, NTE: non-test 
Table 1. Mean and SD of the minimum plateau widths (in $\mathrm{dB}$ ) at $500,1,000,2,000$, and $4,000 \mathrm{~Hz}$ obtained by two methods in the unilateral and bilateral $\mathrm{CHL}$ groups

\begin{tabular}{|c|c|c|c|c|c|}
\hline \multirow{2}{*}{ Study group } & \multirow{2}{*}{ Method } & $500 \mathrm{~Hz}$ & $1,000 \mathrm{~Hz}$ & $2,000 \mathrm{~Hz}$ & $4,000 \mathrm{~Hz}$ \\
\hline & & Mean (SD) & Mean (SD) & Mean (SD) & Mean (SD) \\
\hline \multirow[t]{2}{*}{ Unilateral $\mathrm{CHL}$} & Plateau & $45.34(6.93)$ & $46.03(7.24)$ & $47.59(7.97)$ & $52.24(7.26)$ \\
\hline & Formula & $46.72(7.47)$ & $48.10(6.73)$ & $48.28(7.10)$ & $53.97(6.46)$ \\
\hline \multirow[t]{2}{*}{ Bilateral $\mathrm{CHL}$} & Plateau & 7.33 (8.78) & $8.83(10.39)$ & $22.33(12.84)$ & $14.00(12.34)$ \\
\hline & Formula & $6.67(9.22)$ & $8.67(10.25)$ & $22.50(11.79)$ & $14.17(11.60)$ \\
\hline
\end{tabular}

$\mathrm{CHL}$ : conductive hearing loss, SD: standard deviation

Table 2. Minimum and maximum of the minimum plateau widths (in $\mathrm{dB}$ ) at 500, 1,000, 2,000, and 4,000 $\mathrm{Hz}$ obtained by two methods in the unilateral and bilateral $\mathrm{CHL}$ groups

\begin{tabular}{|c|c|c|c|c|c|c|c|c|c|}
\hline \multirow{2}{*}{ Study group } & \multirow{2}{*}{ Method } & \multicolumn{2}{|c|}{$500 \mathrm{~Hz}$} & \multicolumn{2}{|c|}{$1,000 \mathrm{~Hz}$} & \multicolumn{2}{|c|}{$2,000 \mathrm{~Hz}$} & \multicolumn{2}{|c|}{$4,000 \mathrm{~Hz}$} \\
\hline & & Min & Max & Min & Max & Min & Max & Min & Max \\
\hline \multirow[t]{2}{*}{ Unilateral CHL } & Plateau & 30 & 60 & 30 & 60 & 35 & 65 & 40 & 65 \\
\hline & Formula & 35 & 65 & 35 & 60 & 35 & 70 & 40 & 65 \\
\hline \multirow[t]{2}{*}{ Bilateral CHL } & Plateau & 0 & 30 & 0 & 35 & 0 & 45 & 0 & 40 \\
\hline & Formula & 0 & 35 & 0 & 35 & 0 & 40 & 0 & 35 \\
\hline
\end{tabular}

CHL: conductive hearing loss

Table 3. Standard error differences and significant indexes of the minimum plateau width for two methods at 500, 1,000, 2,000, and $4,000 \mathrm{~Hz}$ in the unilateral and bilateral $\mathrm{CHL}$ groups

\begin{tabular}{|c|c|c|c|c|c|c|c|c|}
\hline \multirow{2}{*}{ Hearing status } & \multicolumn{2}{|c|}{$500 \mathrm{~Hz}$} & \multicolumn{2}{|c|}{$1,000 \mathrm{~Hz}$} & \multicolumn{2}{|c|}{$2,000 \mathrm{~Hz}$} & \multicolumn{2}{|c|}{$4,000 \mathrm{~Hz}$} \\
\hline & S.E. Diff. (dB) & Sig. & S.E. Diff. (dB) & Sig. & S.E. Diff. (dB) & Sig. & S.E. Diff. (dB) & Sig. \\
\hline Unilateral CHL & 1.893 & 0.469 & 1.837 & 0.265 & 1.984 & 0.729 & 1.806 & 0.344 \\
\hline Bilateral CHL & 2.325 & 0.775 & 2.665 & 0.950 & 3.185 & 0.958 & 3.093 & 0.957 \\
\hline
\end{tabular}

CHL: conductive hearing loss

evident in Table 2 data. As seen in this table, the minimum of $\mathrm{mPW}$ is $0 \mathrm{~dB}$ for some people with bilateral $\mathrm{CHL}$, meaning there was no plateau between IML and the maximum amount of NBN in the NTE. In fact, because of the great ABG, NBN intensities from IML to the next were in the overmasking section of the masking curve.

Because of the normal distribution of $\mathrm{mPW}$ data based on the Kolmogorov-Smirnov test, the results at all four frequencies tested with an independent sample t-test showed that the $\mathrm{mPW}$ means were equal for the plateau and formula methods for the unilateral CHL group (Table 3).

Like the data of individuals with unilateral CHL, the $\mathrm{mPW}$ data in bilateral hearing loss group had a normal distribution at all of frequencies (based on the Kolmogorov-Smirnov test), and therefore with implementation of the independent sample $\mathrm{t}$-test, it became clear that there were no significant differences between the mPW means in two methods (Table 3 ).

\section{Discussion}

Yacullo [11] has mentioned that the principles of clinical masking are difficult for many clinicians to understand, and therefore providing a strong foundation with a theoretical and empirical bases of masking is essential for every clinician. The masking diagram is familiar to audiologists and its plateau part is important in clinical masking and is characterized by its width. Audiologists require a 15-20 dB plateau width for clinical applications according to Gelfand's method [9]. The $\mathrm{ABG}_{\mathrm{NTE}}$ affects the width of the plateau: the wider $\mathrm{ABG}$, the narrower the plateau [1]. Since clinical masking is started from IML, knowing the size of the plateau width after it is important. However, it is not necessary to determine the total amount of plateau width in clinical audiometry $[6,10]$. Plateau width is important for at least two reasons: confidence in the masked threshold in the wide plateau and inability to determine the masked threshold in the very narrow plateau [9].

The objective of current study was to determine the width of a segment of a plateau between two noise levels presented to NTE. Regarding the role of IML in clinical masking, we searched for the amount of plateau width at the IML. The data for the unilateral CHL group showed that the average $\mathrm{mPW}$ was almost the same at different frequencies and methods. Although the mPW values were different because of threshold and IA changes at each frequency, there were no significant differences between the plateau search and formula methods at all four frequencies. The $\mathrm{mPW}$ difference between the two methods in the unilateral CHL group was \pm 5 $\mathrm{dB}$ in more than $90 \%$ of cases. 
The difference between the two methods for the average $\mathrm{mPW}$ in the bilateral CHL group was also not significant based on the $95 \%$ confidence interval. The mPW mean in this group was less than previous group. This finding can be explained by Turner's equation $\left(\mathrm{PW}_{\mathrm{AC}}=2 \mathrm{IA}-\mathrm{ABG}_{\mathrm{TE}}-\mathrm{ABG}_{\mathrm{NTE}}\right)$ that was extracted from Liden, et al.'s equation for the minimum effective and maximum usable masking $[6,9]$. $\mathrm{ABG}_{\mathrm{NTE}}$ in the unilateral CHL group is ideally equal to zero but in the bilateral CHL group is greater than zero, and therefore, based on this equation, in the bilateral CHL group in addition to AB$\mathrm{G}_{\mathrm{TE}}, \mathrm{ABG}_{\mathrm{NTE}}$ is also reduced from 2IA and therefore $\mathrm{PW}_{\mathrm{AC}}$ is smaller than the unilateral CHL group. In people with bilateral CHL, the mPW difference between the formula and plateau search method was only $\pm 5 \mathrm{~dB}$ in more than $93 \%$ of cases.

The standard deviation of data for the bilateral CHL participants was higher than that of the unilateral CHL group due to IA and binaural ABG variations. As shown in Table 2, the lowest $\mathrm{mPW}$ obtained at each test frequency was $0 \mathrm{~dB}$, which means there was no plateau after IML. In this situation, $\mathrm{ABG}_{\mathrm{NTE}}$ is large, so it is likely the "Masking Dilemma" described by Naunton [12] had occurred. In fact, during IML delivery, if the previous AC or BC thresholds are repeated, the same thresholds are true and with an increasing noise level, overmasking will happen.

During the test, especially in the unilateral CHL group, sometimes the plateau part was not in a conventional steady state and continued for one further step. This could be due to threshold variability or central masking; an elevation in hearing sensitivity of the TE, as a result of introducing masking noise in the NTE, presumably due to the influence of masking noise on central auditory function $[3,13]$. The purpose of this study was plateau width determining from IML to the beginning of overmasking entry, and therefore, since the formula also computes the width of the plateau, it is expected that the effect of these factors is the same for both methods.

The $\mathrm{mPW}$ mentioned in this paper is equivalent to the adequate masking range (AMR) defined by Turner [10], which is the range of the masking noise level from IML to the maximum usable masking before overmasking $\left(\mathrm{AMR}=\mathrm{BC}_{\mathrm{TE}}+\right.$ IA-IML). Of course, the $\mathrm{mPW}$ can be in any part of plateau of the masking diagram. According to Turner's definition of AMR, for CHL, mPW and AMR are the same. In the other words, at least for $\mathrm{CHL}, \mathrm{AMR}$ is equal to $\mathrm{mPW}: \mathrm{AMR}=\mathrm{mPW}$.

That means:

$$
\begin{aligned}
& {\left[\mathrm{BC}_{\mathrm{TE}}+\mathrm{IA}-\mathrm{IML}\right]=\left[\left(\mathrm{N}_{2}-\mathrm{IML}\right)-\left(\mathrm{T}_{2}-\mathrm{T}_{1}\right)\right]} \\
& \mathrm{IA}=\left(\mathrm{N}_{2}-\mathrm{BC}_{\mathrm{TE}}\right)-\left(\mathrm{T}_{2}-\mathrm{T}_{1}\right)
\end{aligned}
$$

It should be noted that the above equation is true when $\mathrm{N}_{2}$ is equal to or greater than the maximum usable masking. Therefore, the $\mathrm{mPW}$ is equivalent to the width from the maximum usable masking to IML, which is the same as AMR. In fact, based on the results of this study and the mPW formula, the IA value can be estimated by $\pm 5 \mathrm{~dB}$ error in $90 \%$ of cases.

The great advantage of applying the $\mathrm{mPW}$ formula is no actual values of $\mathrm{AC}$ and $\mathrm{BC}$ thresholds in $\mathrm{TE}, \mathrm{ABG}_{\mathrm{NTE}}$ or IA is needed (after initial determination of binaural AC thresholds at the beginning of audiometry, the noise values can be selected for any value in the NTE). Therefore, it is easier to perform and takes less time in clinic. As already mentioned, the $\mathrm{mPW}$ formula has been derived from the masking diagram, and therefore it is possible to use the formula to describe the different sections of the masking diagram (for example, if the mPW value is equal to the noise changes $\left(\mathrm{N}_{2}-\right.$ $\mathrm{N}_{1}$ ), both points are at the plateau and each of the tones represents the threshold). Based on the results of this research, in unilateral hearing loss if masking for AC is needed, after determining the $\mathrm{T}_{1}$ following IML in the NTE, the $\mathrm{N}_{2}$ value can be taken from the difference between maximum output of the audiometer and $T_{1}$ in the TE. If there is not a plateau width required for masking $(15-20 \mathrm{~dB})$, this means there is a profound hearing loss in the TE.

Finally, it should be noted that even when central masking occurs and $\mathrm{N}_{2}$ is not in the overmasking part of the masking diagram, the $T_{2}$ value and thus the $T_{2}-T_{1}$ values will be increased due to central masking and accordingly the $\mathrm{mPW}$ will be smaller. Therefore, the resulting $\mathrm{mPW}$ can be more reliable, especially in situations in which the plateau width decreases.

In conclusion, according to the results of the current study, the formula method can be used for estimating the $\mathrm{mPW}$ between two noise levels in clinical masking for the unilateral and bilateral CHL. Using this method helps audiologists to be aware of 15-20 dB plateau width before starting of traditional plateau method in clinical applications. In fact, threshold obtaining of TE by two tones for two noise levels delivered to the NTE is enough to estimate the $\mathrm{mPW}$ between these two noise points, it is faster and not necessary that the clinician knows actual values of masking diagram components.

\section{Acknowledgments}

This study was part of a Ph.D. Dissertation approved by Iran University of Medical Sciences; Tehran, Iran (Contract number 1395. 9211303207). The cooperation of all participants in this survey is greatly appreciated.

\section{Conflicts of interest}

The authors have no financial conflicts of interest. 


\section{REFERENCES}

1) Martin F, Clark J. Masking. In: Martin F, Clark J, editors. Introduction to audiology. 11th ed. Upper Saddle River (NJ): Pearson Education, Inc:;2012. p.130-52.

2) Valente M. Pure-tone audiometry and masking. San Diego (CA): Plural Publishing, Inc;2009. p.83-116.

3) Stach BA. Comprehensive dictionary of audiology. Baltimore (MD): Williams \& Wilkins;1997. p.39\&54.

4) Studebaker GA. Clinical masking. In: Rintelmann WF, edtor. Hearing assessment. Baltimore (MD): University Park Press;1979. p.51100

5) Liden G, Nilsson G, Anderson H. Masking in clinical audiometry. Acta Otolaryngol 1959;50:125-36.

6) Turner RG. Masking redux. I: an optimized masking method. J Am Acad Audiol 2004;15:17-28.

7) Sanders JW. Masking. In: Katz J, editor. Handbook of clinical au- diology. Baltimore (MD): Williams \& Wilkins;1972. p.111-42.

8) Hood JD. The principles and practice of bone conduction audiometry: a review of the present position. Laryngoscope 1960;70:1211-28.

9) Gelfand SA. Clinical masking. In: Gelfand SA, editor. Essentials of audiology. 4th ed. New York (NY): Thieme Medical Publishers; 2016. p.248-72.

10) Turner RG. Masking redux. II: a recommended masking protocol. J Am Acad Audiol 2004;15:29-46.

11) Yacullo WS. Clinical masking. In: Katz J, Chasin M, English K, Hood LJ, Tillery KL, editor. Handbook of clinical audiology. 7th ed. Philadelphia (PA): Wolters Kluwer Health;2015. p.77-112.

12) Naunton RF. A masking dilemma in bilateral conduction deafness. Arch Otolaryngol 1960;72:753-7.

13) Wegel RL, Lane CE. The auditory masking of one pure tone by another and its probable relation to the dynamics of the inner ear. Phys Rev 1924;23:266-85. 